REFERENCES

[1] Chatzidionysiou, et al. J Rheumatol. 2017 Feb;44(2):162-169.

Disclosure of Interests: None declared DOI: 10.1136/annrheumdis-2019-eular.6215

\section{AB0409 B CELLS PROFILE AS A BIOMARKER FOR EARLY IDENTIFICATION OF OPTIMAL RESPONDERS TO TNF INHIBITORS IN RHEUMATOID ARTHRITIS}

Cristina Sobrino ${ }^{1}$, Borja Hernández-Breijo², Carlota García-Hoz ${ }^{3}$, Israel NietoGañán $^{3}$, Victoria Navarro-Compán ${ }^{2}$, Ana Martínez-Feito ${ }^{2}$, Javier Bachiller-Corral $^{1}$, Gemma Bonilla ${ }^{2}$, Paloma Lapuente-Suanzes ${ }^{3}$, Cristina Pijoan Moratalla ${ }^{1}$, Dora Pascual-Salcedo ${ }^{2}$, Alejandro Balsa ${ }^{2}$, Garbiñe Roy ${ }^{3}$, Mónica Vázquez $^{1}$, Luisa María Villar ${ }^{3}$, Chamaida Plasencia ${ }^{2}$, Eulalia Rodríguez-Martín ${ }^{3} .{ }^{1}$ Rheumatology Department. Ramón y Cajal University Hospital, IRYCIS., Madrid, Spain; ${ }^{2}$ ImmunoRheumatology Research Group, IdiPaz. La Paz University Hospital., Madrid, Spain; ${ }^{3}$ Immunology Department. Ramón y Cajal University Hospital, IRYCIS., Madrid, Spain

Background: The most common biological agents used as disease-modifying treatment in rheumatoid arthritis (RA) are TNF inhibitors (TNFi). Although these new strategies to treat RA have improved the course of the disease, approximately $30-50 \%$ of patients do not respond to this therapy. Early identification of optimal responders is crucial in the clinical setting.

Objectives: The aim of this study was to investigate if baseline percentages of different leukocyte subsets in peripheral blood (PBMCs) can contribute to identify RA patients who will respond to TNFi.

Methods: This was a prospective bi-center pilot study including 100 RA patients under TNFi therapy. Clinical activity was assessed at baseline and 6 months of treatment by disease activity score 28 (DAS28), considering optimal responders if they reached remission at 6 months (DAS28 $\leq 2.6)$. PBMCs were obtained before treatment and different leukocyte subsets were evaluated by flow cytometry (FACSCantoll instrument). The association between the percentage of PBMCs at baseline and clinical response at 6 months was evaluated through logistic regression models (odds ratio; $95 \% \mathrm{Cl}$ ). All the analyses were adjusted by sex, age, disease duration, concomitant methotrexate, baseline DAS28 and seropositivity (ACPA and/or RF).

Results: Demographic characteristics are shown in Table 1. After $6 \mathrm{~m}$ of TNFi treatment, $40 \%$ of the patients achieved clinical remission. A significant association between higher percentage of total $B$ cells $(O R=1.19$; $95 \%$; Cl:1.05-1.35; $\mathrm{p}=0.007$ ) and naive $\mathrm{B}$ cells (Bn; OR=1,32; 95\%; IC:1.08-1.61; $\mathrm{p}=0.007)$ at baseline and clinical response was found. The other PBMC subsets (monocytes, NK cells, CD4+ and CD8+ T cells subtypes) did not show statistical differences (Figure 1).

Conclusion: Our results suggest that basal B cells profile may contribute to identify optimal responders to TNFi in RA. (Funding: ISCIII (PI16/ 01092, Pl16/00474).

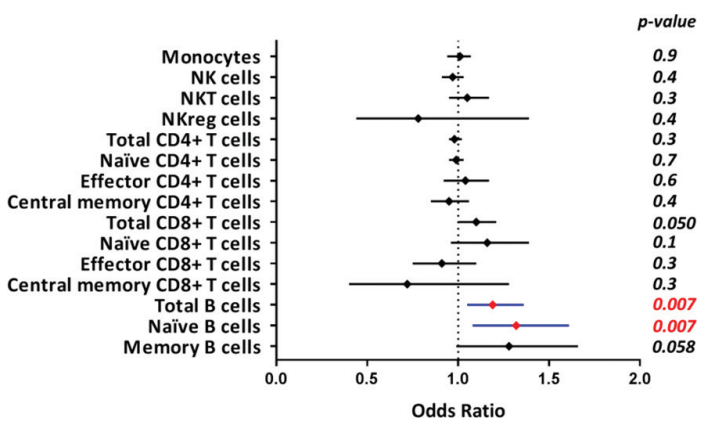

Figure 1. legend: Association between the percentage of PBMC subset before TNFi and the clinical remission. Multivariate logistic regression analysis was performed for each PBMC subset. The percentage of total B cells and in naïve B cells were independently associated with the clinical response. No association was found in other PBMC subsets. The analyses were adjusted by sex, age, disease duration, concomitant-methotrexate, seropositivity and baseline-DAS28.

\begin{tabular}{|c|c|}
\hline Baseline patients' characteristics & Total patients $(n=100)$ \\
\hline Age (years); mean $\pm S D$ & $53 \pm 13$ \\
\hline Female; $\mathbf{n}(\%)$ & $84(84)$ \\
\hline Disease duration (years); median (IQR) & $8(4-12)$ \\
\hline Rheumatoid factor positive; $\mathbf{n}(\%)$ & $77(77)$ \\
\hline ACPA positive; $n$ (\%) & $83(83)$ \\
\hline \multicolumn{2}{|l|}{ Smoking habit; n (\%) } \\
\hline no & $46(46)$ \\
\hline smoker & $20(20)$ \\
\hline ex-smoker & $34(34)$ \\
\hline Body mass index $\left(\mathrm{kg} / \mathrm{m}^{2}\right)$; median (IQR) & $24.8(22.9-29.6)$ \\
\hline DAS28; mean $\pm S D$ & $4.8 \pm 1.2$ \\
\hline CRP (mg/L); median (IQR) & $5.7(2.2-12.4)$ \\
\hline Previous TNFi treatment; $\mathbf{n}(\%)$ & $13(13)$ \\
\hline \multicolumn{2}{|l|}{ TNFi type; $n$ (\%) } \\
\hline Monoclonal antibodies & $55(55)$ \\
\hline Etanercept & $45(45)$ \\
\hline TNFi monotherapy & $4(4)$ \\
\hline Concomitant csDMARD; $\mathrm{n}(\%)$ & $96(96)$ \\
\hline OnlyMethotrexate (MTX) & $51(51)$ \\
\hline OnlyothercsDMARDs $(O D)^{*}$ & $18(18)$ \\
\hline$M T X+O D$ & $27(27)$ \\
\hline Prednisone; $\mathrm{n}(\%)$ & $57(57)$ \\
\hline
\end{tabular}

Table1

Disclosure of Interests: Cristina Sobrino: None declared, Borja Hernández-Breijo: None declared, Carlota García-Hoz: None declared, Israel Nieto-Gañán: None declared, Victoria Navarro-Compán: None declared ANA MARTÍNEZ-FEITO: None declared, Javier Bachiller-Corral: None declared, Gemma Bonilla: None declared, Paloma Lapuente-Suanzes: None declared, Cristina Pijoan Moratalla: None declared, DORA PASCUAL-SALCEDO Grant/research support from: Pfizer, Speakers bureau: Pfizer, Abbvie, Takeda, Alejandro Balsa Grant/research support from: Abbvie, Pfizer, Novartis, BMS, Nordic, Sanofi, Consultant for: Abbvie, Pfizer Novartis, BMS, Nordic, Sanofi, Sandoz, Lilly, Paid instructor for: Pfizer, Speakers bureau: Pfizer, Novartis, UCB, Nordic, Sanofi, Sandoz, Lilly, Garbiñe Roy: None declared, Mónica Vázquez: None declared, Luisa María Villar: None declared, Chamaida Plasencia Speakers bureau: Pfizer, MSD, Eulalia Rodríguez-Martín: None declared

DOI: 10.1136/annrheumdis-2019-eular.4730

\section{AB0410 DISCONTINUATION OF ORAL GLUCOCORTICOID AFTER INITIATION OF BIOLOGICAL DMARD DUE TO A HIGHER DOSE OF METHOTREXATE; A RETROSPECTIVE OBSERVATIONAL STUDY BASED ON DATA FROM A JAPANESE MULTICENTER REGISTRY STUDY}

Mochihito Suzuki, Toshihisa Kojima, Nobunori Takahashi, Naoki Ishiguro. Nagoya University Graduate School of Medicine, Orthopedics, Nagoya, Japan

Background: In the treatment of rheumatoid arthritis, glucocorticoid that provide anti-inflammatory effects in the early stage of treatment is an important drug. We recommend discontinuing of glucocorticoid as much 